\title{
Erratum to: Neutrophil-to-lymphocyte ratio acts as a prognostic factor for patients with advanced hepatocellular carcinoma
}

\author{
Xing $\mathrm{Li}^{1,2} \cdot$ Zhan-Hong Chen ${ }^{2} \cdot$ Xiao-Kun $\mathrm{Ma}^{2} \cdot \mathrm{Jie} \mathrm{Chen}^{2} \cdot$ Dong-Hao $\mathrm{Wu}^{2} \cdot \mathrm{Qu} \mathrm{\textrm {Lin } ^ { 2 }}$ •

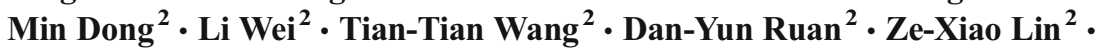 \\ Yan-Fang Xing ${ }^{3} \cdot$ Yun Deng ${ }^{4} \cdot$ Xiang-Yuan $\mathrm{Wu}^{2} \cdot$ Jin-Yun $\mathrm{Wen}^{2}$
}

Published online: 21 July 2016

(C) International Society of Oncology and BioMarkers (ISOBM) 2016

Erratum to: Tumor Biol. (2014) 35:11057-11063

DOI: 10.1007/s13277-014-2360-8

The original version of this article unfortunately contained an error in the affiliation section.

The first author affiliation should have been The Institute of Human Virology, Zhongshan School of Medicine, Sun YatSen University and Department of Medical Oncology, The Third Affiliated Hospital of Sun Yat-sen University not Department of Medical Oncology, The Third Affiliated Hospital of Sun Yat-sen University.

The online version of the original article can be found at http://dx.doi.org/ 10.1007/s13277-014-2360-8.

Xiang-Yuan $\mathrm{Wu}$

wuxiangy@mail.sysu.edu.cn

$\triangle$ Jin-Yun Wen

wenjingy@mail.sysu.edu.cn

1 The Institute of Human Virology, Zhongshan School of Medicine, Sun Yat-Sen University, Guangzhou, People's Republic of China

2 Department of Medical Oncology, The Third Affiliated Hospital of Sun Yat-sen University, 600 Tianhe Road, Guangzhou 510630,

People's Republic of China

3 Department of Nephrology, The Third Affiliated Hospital of Guangzhou Medical University, 63 Duobao Road, Guangzhou 510150, People's Republic of China

4 Department of Radiation Oncology, The Fifth Affiliated Hospital of Sun Yat-sen University, 52 Meihua Dong Road, Zhuhai, People's Republic of China 